\title{
Quantum theory of spontaneous and stimulated emission of surface plasmons
}

\author{
Alexandre Archambault, François Marquier, and Jean-Jacques Greffet \\ Laboratoire Charles Fabry, Institut d Optique, CNRS-Univ Paris-Sud, Campus Polytechnique, RD128, 91127 Palaiseau Cedex, France
}

\author{
Christophe Arnold \\ Laboratoire de Photonique et de Nanostructures, CNRS, Route de Nozay, 91460 Marcoussis, France
}

(Received 20 April 2010; revised manuscript received 11 June 2010; published 12 July 2010)

\begin{abstract}
We introduce a quantization scheme that can be applied to surface waves propagating along a plane interface. An important result is the derivation of the energy of the surface wave for dispersive nonlossy media without invoking any specific model for the dielectric constant. Working in Coulomb's gauge, we use a modal representation of the fields. Each mode can be associated with a quantum harmonic oscillator. We have applied the formalism to derive quantum mechanically the spontaneous emission rate of surface plasmon by a twolevel system. The result is in very good agreement with Green's tensor approach in the nonlossy case. Green's approach allows also to account for losses, so that the limitations of a quantum approach of surface plasmons are clearly defined. Finally, the issue of stimulated versus spontaneous emission has been addressed. Because of the increasing density of states near the asymptote of the dispersion relation, it is quantitatively shown that the stimulated emission probability is too small to obtain gain in this frequency region.
\end{abstract}

DOI: 10.1103/PhysRevB.82.035411 PACS number(s): 03.70.+k, 73.20.Mf, 78.20.Bh, 78.45. $+\mathrm{h}$

\section{INTRODUCTION}

Quantum theory of light is a useful tool to describe microscopic interactions between light and matter. The electromagnetic state is represented by photon number states and the electromagnetic field becomes an operator. ${ }^{1}$ Such a description of light provides a quantitative description of absorption, spontaneous and stimulated emission of photons by a two-level system. In particular, it allows to derive a quantitative treatment of light amplification. It also predicts pure quantum effects, such as photon coalescence or antibunching. Quantum theory of light can be extended to nondispersive and nonlossy media. Each photon in the material corresponds to the excitation of a mode characterized by a wave vector $\mathbf{k}$ and circular frequency $\omega$, such as $k=n \omega / c$, where $n$ is the refractive index of the medium and $c$ the light velocity in a vacuum. It is the purpose of this paper to introduce a quantification scheme for surface waves propagating along an interface.

It is well known that electromagnetic surface waves called surface plasmons exist at interfaces between metals and dielectrics. ${ }^{2}$ Their quantum nature has been demonstrated by energy-loss spectroscopy experiments on thin metallic films reported by Powell and Swan. ${ }^{3}$ Single optical plasmons have been excited recently along a metallic nanowire ${ }^{4,5}$ Surface plasmons are associated with collective oscillation of free electrons in the metal at the surface. Similar electromagnetic fields exist also on polar materials and are called surface phonon polariton. Both surface-plasmon-polaritons and surface-phonon-polaritons propagate along the interface and decrease in the direction perpendicular to the surface. Such a resonance is therefore called surface wave in a more general way. Most studies deal with a plane interface between air or vacuum and a nonlossy material. In this case, it is well known $^{2}$ that a surface wave can exist if the dielectric constant $\epsilon(\omega)$ has a real part lower than -1 .

Losses are often a serious limitation for many practical applications envisioned for surface plasmons. This problem could be circumvented by introducing gain in the system. Studies have been made in such a way with metallic nanoparticles embedded in a gain medium both numerically with dye molecules ${ }^{6}$ or quantum $\operatorname{dots}^{7,8}$ and experimentally. ${ }^{9}$ Seidel et al. ${ }^{10}$ reported the first experiment demonstrating the amplification of surface plasmons on a flat silver film surrounded by a solution of dye molecules. Since then, a few studies have dealt with stimulated emission of surface plasmons on flat interfaces both experimentally ${ }^{11,12}$ and theoretically. ${ }^{13}$ Such works have paved the way to active plasmonics $^{14-16}$ and nanolasers ${ }^{17,18}$ or more precisely to spasers ${ }^{8,19}$ or surface-plasmon amplification by stimulated emission introduced by Bergman and Stockman and demonstrated experimentally recently. ${ }^{20,21}$

It is clear that a quantum treatment of surface plasmon could be useful for many applications. For instance, an efficient single-photon emitter could be optimized. ${ }^{22}$ A quantum treatment allows to model stimulated emission and therefore to specify gain conditions and laser operation. It could also allow to analyze pure quantum effects for surface plasmons such as single-plasmon interferences, quantum correlations, ${ }^{23}$ bunching, strong-coupling regime ${ }^{24,25}$ or single-photon excitation of surface plasmon. ${ }^{26-29}$ The first quantization scheme for surface plasmon on a metallic surface has been reported by Elson and Ritchie. ${ }^{30}$ In their work, the metal is characterized by a nonlossy Drude model so that real optical properties cannot be included. Using Green's approach, Gruner and Welsch ${ }^{31}$ introduce a quantization scheme for electromagnetic fields in dispersive and absorptive materials. It should hence be possible to quantize the field associated with surface waves using their model. Note that due to losses, they cannot obtain operators for modes but only local operators: one recovers the usual creation/ annihilation operators in the limit of zero losses. A related work, reported in the early nineties by Babiker et al. ${ }^{32}$ dealt with the quantization of interface optical phonons in quantum well, which could appear also as a confined surface phonon in a heterostructure. 


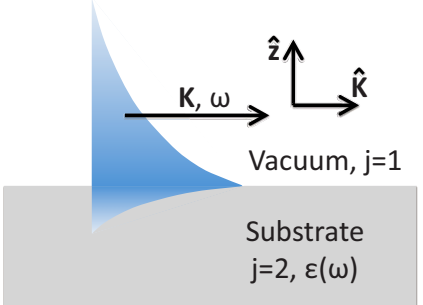

FIG. 1. (Color online) Surface wave on a plane interface. The surface mode is characterized by its circular frequency $\omega$ and the projection of the wave vector along the interface $\mathbf{K}$. $\hat{\mathbf{K}}$ and $\hat{\mathbf{z}}$ are unit vectors along and perpendicular to the plane interface, respectively.

In this paper, a quantization scheme that is not based on a specific model of the dielectric constant is introduced. The aim is to quantize the field by accounting for the experimental dispersion properties of the medium. The procedure follows the quantization scheme for photons in a vacuum. We will first introduce a classical-mode description of the surface waves and discuss the dispersion relation. A key issue for quantization is the definition of the energy of surface waves for dispersive lossy media. The problem of electromagnetic energy in a dispersive and lossy medium has been recently addressed in a paper by Stallinga. ${ }^{33}$ The third section addresses the problem of the electromagnetic energy associated with surface waves in a simpler case following Landau and Lifchitz for nonlossy dielectric material. ${ }^{34}$ The quantization scheme is finally described in the fourth section. In order to check our results, we apply our formalism in the fifth section to the calculation of the spontaneous emission of a two-level system in the presence of surface plasmons. The Purcell factor (i.e., the local density of states normalized by the vacuum density of states) and Einstein's coefficients are also derived using this model.

\section{MODAL DESCRIPTION OF SURFACE WAVES}

Let us consider surface waves propagating on a plane interface at $z=0$ separating two semi-infinite media (Fig. 1). One of them is a vacuum or air and the second is a metal or a polar material. A surface mode is characterized by its circular frequency $\omega$ and the projection of the wave vector $\mathbf{K}$ on the plane perpendicular to the $z$ axis. The material has a dielectric constant $\epsilon(\omega)$. We use Coulomb's gauge $[\operatorname{div} \mathbf{A}(\mathbf{r}, t)=0]$ to write the magnetic and electric fields

$$
\begin{aligned}
& \mathbf{B}(\mathbf{r}, t)=\nabla \times \mathbf{A}(\mathbf{r}, t), \\
& \mathbf{E}(\mathbf{r}, t)=-\frac{\partial \mathbf{A}(\mathbf{r}, t)}{\partial t} .
\end{aligned}
$$

The field produced by any distribution of sources in the presence of an interface can be computed using Green's tensor. By extracting the pole contribution, it is possible to derive the general form of the surface-plasmon field. The details of this procedure can be found in Ref. 35. The corresponding vector potential can be cast in the form

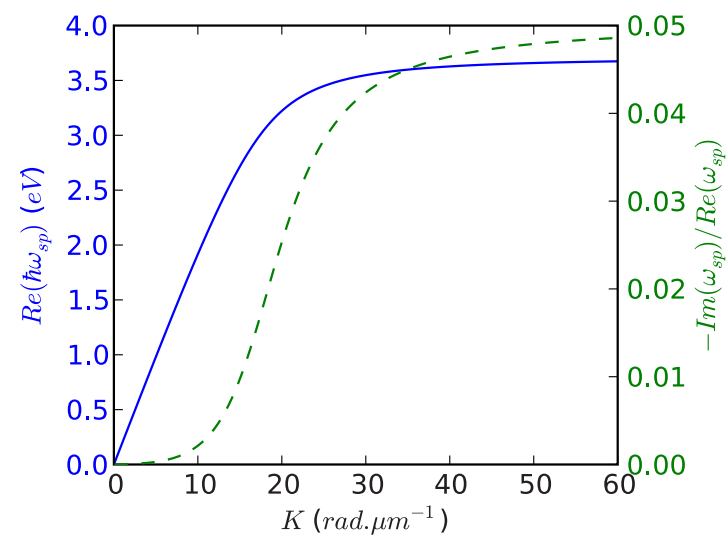

FIG. 2. (Color online) Dispersion relation of a surface plasmon on a plane interface between air and silver (solid line, left axis) and variation in the imaginary part of $\omega$ (dashed line, right axis). The dispersion relation has been obtained using the silver dielectric constant given in Appendix A.

$$
\mathbf{A}(\mathbf{r}, t)=\int \frac{d^{2} \mathbf{K}}{(2 \pi)^{2}} \alpha_{\mathbf{K}} \mathbf{u}_{\mathbf{K}}(z) \exp (i \mathbf{K} \cdot \mathbf{r}) \exp \left(-i \omega_{s p} t\right)+\text { c.c. }
$$

where c.c. stands for complex conjugate. In this equation, $\mathbf{K}$ is a real wave vector parallel to the interface and the circular frequency $\omega_{s p}$ is a complex root of the equation

$$
K=\frac{\omega}{c} \sqrt{\frac{\epsilon(\omega)}{\epsilon(\omega)+1}} .
$$

The term $\alpha_{\mathbf{K}}$ is an amplitude associated with wave vector $\mathbf{K}$ in the decomposition. The vectors $\mathbf{u}_{\mathbf{K}}(z)$ are given by

$$
\mathbf{u}_{\mathbf{K}}(z)=\frac{1}{\sqrt{L\left(\omega_{s p}\right)}} \exp \left(i \gamma_{j} z\right)\left(\hat{\mathbf{K}}-\frac{K}{\gamma_{j}} \hat{\mathbf{z}}\right),
$$

where $L\left(\omega_{s p}\right)$ has the dimension of a length and will be fixed later by Eq. (B5) to normalize the energy of each mode. $\gamma_{j}$ is the projection of the wave vector along the $z$ axis, $j=1$ in the region $z>0$, and $j=2$ in the region $z<0$, so that $\gamma_{j}^{2}$ $=\epsilon_{j}\left(\omega_{s p}\right) \omega_{s p}^{2} / c^{2}-K^{2}$. The sign of $\gamma_{j}$ is then chosen such as the field goes to zero when $z$ goes to $\pm \infty$. Let us note that in the nonlossy case, $\gamma_{1}$ and $\gamma_{2}$ are purely imaginary, so that the electric field decays exponentially along the $z$ axis. $\hat{\mathbf{K}}$ and $\hat{\mathbf{z}}$ are unit vectors directed along $\mathbf{K}$ and the $z$ axis, respectively.

Figure 2 shows the dispersion relation of surface plasmons as well as the variation in the imaginary part of the frequency with $K$ on a plane interface of silver. To perform the calculations when a complex frequency is needed, it has been useful to fit the experimental values of the dielectric constant $\epsilon(\omega)$ given by Ref. 36 with an analytical model. The real part of the silver dielectric constant is very well represented by a Drude model given in Ref. 14. For the imaginary part we add to this Drude model a conductivity term, so that the modelized dielectric constant is in very good agreement with the experimental data. The model we used is given in Appendix A. In this example, $\operatorname{Im}\left(\omega_{s p}\right)$ is small, less than $5 \%$, comparing to $\operatorname{Re}\left(\omega_{s p}\right)$. In other words, the lifetime of the 
surface mode is long enough to have a few tens of periods for the oscillating electromagnetic field on the asymptotic part of the dispersion relation and hundreds of periods on the linear part, close to the light cone. Note that this point seems to be rather general. Indeed we found similar ratios for many other materials supporting surface waves.

From the dispersion relation, it is possible to derive the density of states. To this aim, it is convenient to introduce a virtual box, which is in fact a virtual square in the $x-y$ plane of sides $L_{x}$ and $L_{y}$ and size $S=L_{x} \times L_{y}$. Born-Von Karman's conditions yields a quantized value of the wave vector $K_{x}$ $=n_{x} 2 \pi / L_{x}, K_{y}=n_{y} 2 \pi / L_{y}$, where $n_{x, y}$ are relative integers. Let us note that a different expansion of the surface-plasmon field can be used with a complex wave vector and a real frequency as discussed in Ref. 35. We stress here that the Born-von Karman procedure imposes a real wave vector. It follows that the relevant dispersion relation has no backbending as seen in Fig. 2. The reader is referred to Ref. 35 for more details. Substituting the discrete sum $\frac{1}{S} \Sigma_{\mathbf{K}}$ over the quantized wave vector $\mathbf{K}$ and the discrete amplitude $S A_{\mathbf{K}}$ to $\int \frac{d^{2} \mathbf{K}}{(2 \pi)^{2}}$ and $\alpha_{\mathbf{K}}$, respectively, the vector potential can be cast as

$$
\mathbf{A}(\mathbf{r}, t)=\sum_{\mathbf{K}} A_{\mathbf{K}} \mathbf{u}_{\mathbf{K}}(z) \exp (i \mathbf{K} \cdot \mathbf{r}) \exp (-i \omega t)+\text { c.c. },
$$

where we have omitted the subscript $s p$ for the circular frequency $\omega$. We can insert this form in Eqs. (1) and (2) to obtain the electric and magnetic fields. Introducing the notations $\mathbf{k}_{j}=\mathbf{K}+\gamma_{j} \hat{\mathbf{z}}$ and $\mathbf{b}_{\mathbf{K}}(z)=\mathbf{k}_{j} \times \mathbf{u}_{\mathbf{K}}(z)$, we have

$$
\begin{gathered}
\mathbf{E}(\mathbf{r}, t)=i \sum_{\mathbf{K}} \omega A_{\mathbf{K}} \mathbf{u}_{\mathbf{K}}(z) \exp (i \mathbf{K} \cdot \mathbf{r}) \exp (-i \omega t)+\text { c.c. } \\
\mathbf{B}(\mathbf{r}, t)=i \sum_{\mathbf{K}} A_{\mathbf{K}} \mathbf{b}_{\mathbf{K}}(z) \exp (i \mathbf{K} \cdot \mathbf{r}) \exp (-i \omega t)+\text { c.c. }
\end{gathered}
$$

\section{ENERGY OF A SURFACE WAVE}

The quantization procedure is based on the fact that the energy of the field has the structure of a sum of harmonic oscillators. It is thus a key issue to derive the energy of the surface-plasmon field. In this section, we give a brief outline of the derivation and leave the details to Appendix B and supplementary material. ${ }^{37}$ In a vacuum, the energy density is given by ${ }^{38}$

$$
u_{1}=\frac{\epsilon_{0}}{2} \mathbf{E}^{2}(\mathbf{r}, t)+\frac{1}{2 \mu_{0}} \mathbf{B}^{2}(\mathbf{r}, t) .
$$

The electromagnetic energy in a lossy dispersive material is a more subtle issue. This problem has been addressed for the first time by Brillouin. ${ }^{39} \mathrm{He}$ considered a very simple case, with two perfectly monochromatic waves in the material. Landau and Lifchitz ${ }^{34}$ analyzed the energy of an electromagnetic field in a nonlossy dispersive medium, whose frequencies form a narrow continuum around the mean frequency $\omega_{0}$. They dealt with fields such as $\mathbf{E}=\mathbf{E}_{0}(t) \exp ($ $\left.-i \omega_{0} t\right), \mathbf{E}_{0}(t)$ varying slowly over the period $2 \pi / \omega_{0}$. In the Appendix B, we follow this method. The main idea is to derive the work done by an external operator to build adiabatically the field amplitude. This work is equal to the total amount of electromagnetic energy of the surface waves for a nonlossy medium. Note that more recently, Stallinga ${ }^{33}$ derived an expression of the energy for dispersive and lossy materials. The result is the same provided that $\epsilon$ is replaced by $\operatorname{Re}(\epsilon)$. This suggests that it is possible to neglect losses in the calculation of the energy. Actually, it is essential to deal with a nonlossy medium to have well-defined modes. A key issue regarding this approximation is whether the dispersion relation is modified by the presence of losses. Indeed, the density of states critically depends on the dispersion relation. We compared the dispersion relation obtained using $\operatorname{Re}\left(\omega_{s p}\right)$ for a lossy medium with the dispersion relation with a nonlossy medium in the case of silver. We found a relative difference between the two dispersion relations always less than $1.5 \times 10^{-3}$.

We will thus neglect the losses of the medium in the derivation of the energy. The calculation outlined in Appendix B gives the total energy of the surface waves

$$
U=\sum_{\mathbf{K}} \epsilon_{0} \omega^{2} S\left[A_{\mathbf{K}} A_{\mathbf{K}}^{*}+A_{\mathbf{K}}^{*} A_{\mathbf{K}}\right]
$$

We emphasize that this convenient expression for the energy is obtained using the right normalization condition on $L(\omega)$ or equivalently on $\mathbf{u}_{\mathbf{K}}(z)$ given, respectively, by Eqs. (B5) and (B6).

\section{QUANTIZATION OF SURFACE WAVES}

We now turn to the quantization of the electromagnetic field of surface plasmons. We first notice that the expression $\epsilon_{0} \omega^{2} S\left[A_{\mathbf{K}} A_{\mathbf{K}}^{*}+A_{\mathbf{K}}^{*} A_{\mathbf{K}}\right]$ of the energy for each mode $\mathbf{K}$, has the structure of the energy of a harmonic oscillator, hence the quantized hamiltonian

$$
\hat{H}=\sum_{\mathbf{K}} \frac{\hbar \omega}{2}\left[\hat{a}_{\mathbf{K}} \hat{a}_{\mathbf{K}}^{\dagger}+\hat{a}_{\mathbf{K}}^{\dagger} \hat{a}_{\mathbf{K}}\right]
$$

with the equivalence

$$
\begin{gathered}
A_{\mathbf{K}} \rightarrow \sqrt{\frac{\hbar}{2 \epsilon_{0} \omega S}} \hat{a}_{\mathbf{K}}, \\
A_{\mathbf{K}}^{*} \rightarrow \sqrt{\frac{\hbar}{2 \epsilon_{0} \omega S}} \hat{a}_{\mathbf{K}}^{\dagger} .
\end{gathered}
$$

The surface wave field is thus quantized by association of a quantum-mechanical harmonic oscillator to each mode $\mathbf{K}$. We introduce $\hat{a}_{\mathbf{K}}^{\dagger}$ and $\hat{a}_{\mathbf{K}}$ which are, respectively, the creation and annihilation operators for the mode $\mathbf{K}$. As in the harmonic-oscillator theory, $\hat{a}_{\mathbf{K}}^{\dagger}$ and $\hat{a}_{\mathbf{K}}$ act on surface wavenumber states $\left|n_{\mathbf{K}}\right\rangle$ which are eigenvectors associated with eigenvalues $\left(n_{\mathbf{K}}+1 / 2\right) \hbar \omega$ of the Hamiltonian $\left(n_{\mathbf{K}}\right.$ is an integer). Operators $\hat{a}_{\mathbf{K}}^{\dagger}$ (respectively, $\hat{a}_{\mathbf{K}}$ ) allow to create (respectively, destroy) a quantum of energy $\hbar \omega$ according to the operating rules ${ }^{1}$ 


$$
\begin{gathered}
\hat{a}_{\mathbf{K}}^{\dagger}\left|n_{\mathbf{K}}\right\rangle=\sqrt{n_{\mathbf{K}}+1}\left|n_{\mathbf{K}}+1\right\rangle, \\
\hat{a}_{\mathbf{K}}\left|n_{\mathbf{K}}\right\rangle=\sqrt{n_{\mathbf{K}}}\left|n_{\mathbf{K}}-1\right\rangle .
\end{gathered}
$$

Different surface modes are independent so that their associated operators commute

$$
\left[\hat{a}_{\mathbf{K}}, \hat{a}_{\mathbf{K}^{\prime}}^{\dagger}\right]=\delta_{\mathbf{K}, \mathbf{K}^{\prime}} .
$$

We can now write the fields as operators acting on the surface-plasmon number quantum states $\left|n_{\mathbf{K}}\right\rangle$

$$
\hat{\mathbf{E}}(\mathbf{r}, t)=i \sum_{\mathbf{K}} \sqrt{\frac{\hbar \omega}{2 \epsilon_{0} S}} \mathbf{u}_{\mathbf{K}}(z) \hat{a}_{\mathbf{K}} \exp (i \mathbf{K} \cdot \mathbf{r}) \exp (-i \omega t)+\text { H.c. },
$$

$$
\hat{\mathbf{B}}(\mathbf{r}, t)=i \sum_{\mathbf{K}} \sqrt{\frac{\hbar}{2 \epsilon_{0} \omega S}} \mathbf{b}_{\mathbf{K}}(z) \hat{a}_{\mathbf{K}} \exp (i \mathbf{K} \cdot \mathbf{r}) \exp (-i \omega t)+\text { H.c. },
$$

where H.c. denotes the hermitian conjugate.

\section{EMISSION RATES: COMPARISON WITH THE CLASSICAL CASE, EINSTEIN'S COEFFICIENTS}

\section{A. Spontaneous emission of a dipole above a metallic interface}

The quantization scheme that we have introduced allows to derive an expression of the electromagnetic field using operators. Hence, we can write interaction hamiltonians and describe the coupling between light and matter. In order to test this quantization procedure, we performed the calculation of the lifetime of a two-level system placed in the vicinity of a metal-vacuum interface so that surface plasmons can be excited. This result is interesting as the lifetime can also be computed using a classical approach as shown, for instance, by Ford and Weber. ${ }^{40}$ More specifically, they showed how to find the surface-plasmon contribution to the lifetime by extracting the pole contribution. By comparing both results, we can assess the validity of the quantum theory of surface plasmon within the approximation of a dispersive but nonlossy medium.

\section{Quantum calculation}

In the quantum approach, we first derive the decay rate associated to the spontaneous emission of surface plasmons of a two-level quantum system close to an interface, using Fermi's golden rule. This gives the surface-plasmon spontaneous emission rate as a function of the matrix element $\langle 2|\hat{\mathbf{D}}| 1\rangle=\mathbf{D}_{12}$ of the dipole moment operator $\hat{\mathbf{D}}$. The details of the calculation are given in Appendix C.

We obtain the following expression for the spontaneous emission rate:

$$
\gamma_{\text {spont }}\left(\mathbf{D}_{12}, \omega_{0}, z\right)=\frac{\omega_{0}\left|\mathbf{D}_{\mathbf{1 2}}\right|^{2}}{2 \epsilon_{0} \hbar} K \frac{d K}{d \omega} \frac{1}{L_{e f f}\left(z, \mathbf{d}_{12}, \omega_{0}\right)}
$$

in which $\mathbf{d}_{12}=\mathbf{D}_{12} /\left|\mathbf{D}_{12}\right|$ is the (possibly complex) polarization of the dipole, $d_{12, z}=\mathbf{d}_{12} \cdot \hat{\mathbf{z}}, \mathbf{d}_{12, \|}=\mathbf{d}_{12}-d_{12, z} \hat{\mathbf{z}}$. We intro-

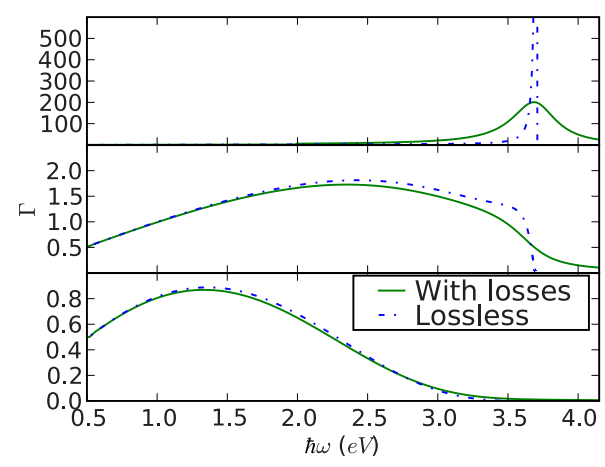

FIG. 3. (Color online) Normalized emission rate $F_{P}$ of a vertical dipole located at $10 \mathrm{~nm}$ (top), $75 \mathrm{~nm}$ (center), and $250 \mathrm{~nm}$ (bottom) from the surface when taking into account losses (solid lines) comparing to the nonlossy cases (dashed-dotted lines).

duced the effective length of the surface-plasmon mode $L_{e f f}\left(z, \mathbf{d}_{12}, \omega_{0}\right)$,

$$
\frac{1}{L_{e f f}\left(z, \mathbf{d}_{12}, \omega_{0}\right)}=\frac{\exp \left(2 i \gamma_{1} z\right)}{L\left(\omega_{0}\right)}\left[\frac{1}{2}\left|\mathbf{d}_{12, \|}\right|^{2}-\epsilon\left(\omega_{0}\right)\left|d_{12, z}\right|^{2}\right] \text {. }
$$

It will be seen later that this length allows to define an effective volume of the plasmon mode.

For comparison with the classical calculation, we normalize $\gamma_{\text {spont }}\left(\mathbf{D}_{12}, \omega_{0}, z\right)$ with the spontaneous emission rate of the same two-state quantum system in a vacuum, given by ${ }^{1}$ $\gamma_{\text {spont }}^{0}=\frac{\omega_{0}^{3}\left|\mathbf{D}_{12}\right|^{2}}{3 \pi \epsilon_{0} \hbar c^{3}}$. This gives the Purcell factor associated to the emission of surface plasmons

$$
F_{P}\left(\mathbf{d}_{12}, \omega_{0}, z\right)=\frac{3 \pi c^{3}}{2 \omega_{0}^{2}} K \frac{d K}{d \omega} \frac{1}{L_{e f f}\left(z, \mathbf{d}_{12}, \omega_{0}\right)}
$$

which does not depend anymore on the amplitude of $\mathbf{D}_{12}$, but only on its polarization $\mathbf{d}_{12}$, its frequency $\omega_{0}$, and its distance to the interface $z$. As expected, the Purcell factor decreases exponentially as the dipole goes farther from the interface, and can have rather high values (see Fig. 3 and comments below) as $\omega_{0}$ gets closer to the asymptotic frequency of surface plasmons if the dipole is not too far from the interface.

This Purcell factor can also be cast under the form

$$
F_{P}\left(\mathbf{d}_{12}, \omega_{0}, z\right)=\omega_{0} g\left(\omega_{0}\right) \frac{\lambda_{0}^{3}}{V_{e f f}\left(z, \mathbf{d}_{12}, \omega_{0}\right)} \frac{3}{8 \pi},
$$

where the (global) density of states of surface plasmons $g(\omega)$ is given by $g(\omega)=S \frac{K}{2 \pi} \frac{d K}{d \omega}$ and $V_{e f f}\left(z, \mathbf{d}_{12}, \omega_{0}\right)$ $=S L_{e f f}\left(z, \mathbf{d}_{12}, \omega_{0}\right)$ is the volume of the surface-plasmon modes of frequency $\omega_{0}$ for a dipole polarization $\mathbf{d}_{12}$ in which the emission occurs. Equation (21) is thus similar to the Purcell factor $F_{P}$ of a dipole interacting with a single-damped mode. ${ }^{41}\left[F_{P}=Q \frac{\lambda^{3}}{V} \frac{3}{4 \pi^{2}}\right.$, or equivalently $F_{P}=\omega g(\omega) \frac{\lambda^{3}}{V} \frac{3}{8 \pi}$ using the density of states of the single mode at resonance, $g(\omega)$ $\left.=\frac{2}{\pi} \frac{Q}{\omega}\right]$.

When dealing with an isotropic distribution of dipoles, the average of the rate of spontaneous emission [Eq. (18)], over the orientations of the dipole $\mathbf{D}_{12}$, should be considered. Let us first introduce the total effective length of the surface- 
plasmon mode, defined as the inverse of the average of $\frac{1}{L_{e f f}\left(z, \mathbf{d}_{12}, \omega_{0}\right)}$ over the directions of $\mathbf{d}_{12}$

$$
\frac{1}{L_{e f f, t o t a l}\left(z, \omega_{0}\right)}=\frac{1}{3} \frac{\exp \left(2 i \gamma_{1} z\right)}{L\left(\omega_{0}\right)}\left[1+\left|\epsilon\left(\omega_{0}\right)\right|\right] .
$$

Calculating the averaged rate of spontaneous emission then amounts to replacing $L_{e f f}\left(z, \mathbf{d}_{12}, \omega_{0}\right)$ by $L_{e f f, t o t a l}\left(z, \omega_{0}\right)$ in Eq. (18)

$$
\gamma_{\text {spont }, \text { total }}\left(\left|\mathbf{D}_{12}\right|, \omega_{0}, z\right)=\frac{\omega_{0}\left|\mathbf{D}_{\mathbf{1 2}}\right|^{2}}{2 \epsilon_{0} \hbar} K \frac{d K}{d \omega} \frac{1}{L_{e f f, t o t a l}\left(z, \omega_{0}\right)} .
$$

More details are given in Appendix C.

\section{Classical approach}

In the previous section, we considered a two-level quantum system having a given polarization $\mathbf{d}_{12}$ and Bohr circular frequency $\omega_{0}$, and we normalized its spontaneous emission rate by its value in a vacuum. The power radiated by a classical harmonic dipole having the same polarization $\mathbf{d}_{12}$ and a circular frequency $\omega_{0}$ can also be normalized by its value in a vacuum. Both expressions give the normalized local density of states, which is a classical quantity. They are therefore equal, that is the normalized radiated power gives the normalized spontaneous emission rate. The normalized radiated power can be expressed as a function of Green's tensor $\overleftrightarrow{\mathbf{G}}\left(\mathbf{r}, \mathbf{r}^{\prime}, \omega\right)$ of the system

$$
F_{P, c l}\left(\mathbf{d}_{12}, \omega_{0}, z\right)=\frac{6 \pi c}{\omega_{0}} \operatorname{Im}\left[\mathbf{d}_{12}^{*} \cdot \stackrel{\leftrightarrow}{\mathbf{G}}\left(z \hat{\mathbf{z}}, z \hat{\mathbf{z}}, \omega_{0}\right) \mathbf{d}_{12}\right] .
$$

Following the steps detailed in Ref. 35, the pole contribution $\overleftrightarrow{\mathbf{G}}_{s p}$ of Green's tensor of a plane interface can be derived, and inserted in Eq. (24). We use here the pole contribution of the surface plasmon with a complex frequency (see Ref. 35). The details of the calculation are given in Appendix D. One finds for the normalized radiated power in the nonlossy case

$$
\begin{aligned}
F_{P, c l}\left(\mathbf{d}_{12}, \omega_{0}, z\right)= & \frac{3 \pi c^{3}}{\omega_{0}^{3}} K^{3} \frac{d K}{d \omega} R\left(K, \omega_{0}\right) \exp \left(2 i \gamma_{1} z\right) \\
& \times\left[\frac{1}{2}\left|\mathbf{d}_{12, \|}\right|^{2}-\epsilon\left(\omega_{0}\right)\left|d_{12, z}\right|^{2}\right] .
\end{aligned}
$$

Comparing Eq. (25) with Eq. (20), one can check $^{37}$ that $F_{P}\left(\mathbf{d}_{12}, \omega_{0}, z\right)=F_{P, c l}\left(\mathbf{d}_{12}, \omega_{0}, z\right)$. We thus recover the quantum spontaneous emission rate in the nonlossy limit of the above classical approach. This result is not surprising. Indeed the normalized spontaneous emission rate yields the local density of states. The latter is a classical quantity. In the quantum approach, it has been calculated using the dispersion relation. In the classical approach, it has been calculated using the Green's tensor. We have thus checked that the mode approach and the Green's formalism approach are equivalent. We now go one step further and compare the quantum approach (without losses) with the Green's tensor approach that accounts for losses. We compute the spontaneous emission rate for both cases in order to assess the role of losses.

\section{Comparison with the lossy case}

The lossy and nonlossy emission rates are compared using Eqs. (20) and (D8). The result is seen on Fig. 3 for a dipole located at three different distances of a silver surface $(10,75$, and $250 \mathrm{~nm})$. It appears that the differences between both curves in this case are still very small as long as the frequency is not too close from the asymptote of the dispersion relation. This is not surprising considering the fact that at this asymptotic value the losses are the most important. Moreover, when the distance between the dipole and the interface increases, the part of the electromagnetic field due to the higher surface-plasmons wave vector decreases, so that the part due to the surface plasmons lying on the linear part of the dispersion relation is more important. As seen in Fig. 2, these surface waves have less losses and the quantum approach is thus more accurate. It follows the important conclusion that the nonlossy medium approximation in the quantum treatment is reasonable to deal with surface waves provided that the frequency is not too close to the asymptotic value.

Note that Ref. 40 provides an expression for the surfaceplasmon emission rate of a dipole close to an interface, which can be compared to ours (details not given here). In the nonlossy case, it can be proved analytically that their results lead to the same normalized emission rate as Eq. (20). In the lossy case, one finds a normalized emission rate close to the one used here [Eq. (D8)], although they are not rigorously equal. Our method gives an expression of the normalized surface-plasmon emission rate as a sum over the modes $\mathbf{K}$ [see Eq. (D4)], which provides a better understanding of the difference between the lossy and the nonlossy cases.

\section{B. Einstein's coefficients}

A quantum approach for surface waves allows us also to derive easily Einstein's coefficients for spontaneous and stimulated emission. The same example of a dipole above the interface is taken. Once again, it is possible to follow the approach described for photons in Ref. 1, for instance. Einstein's coefficient for surface-plasmon spontaneous emission has already been calculated: $A_{21}=\gamma_{\text {spont }}\left(\mathbf{D}_{12}, \omega_{0}, z\right)$ [see Eq. (18)]. In order to obtain Einstein's coefficient for stimulated emission, one needs to start from Eq. (C3). In this equation, the term proportional to $n_{\mathbf{K}}$ is the matrix element for stimulated emission. We note $\langle W(\omega)\rangle$ the energy density of the radiation per unit surface and we assume that it varies slowly for frequencies near $\omega_{0}$. The total energy in the single mode $n_{\mathbf{K}}$ is now replaced by

$$
n_{\mathbf{K}} \hbar \omega \rightarrow S \int d \omega\langle W(\omega)\rangle
$$

The transition rate due to stimulated emission can thus be written 


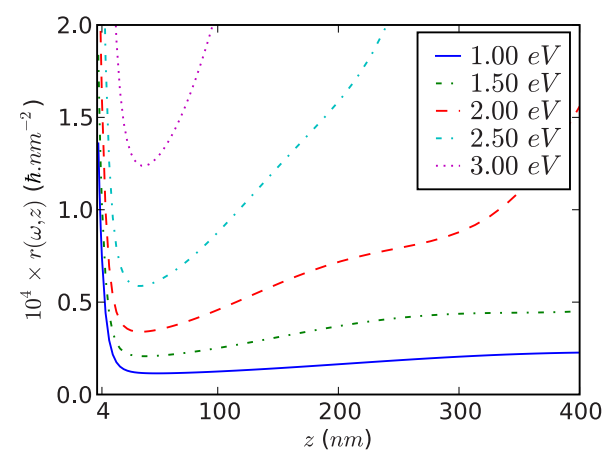

FIG. 4. (Color online) Ratio of Einstein's coefficients $r(\omega, z)$ for (total) spontaneous emission and stimulated emission of surface plasmons as a function of the distance to the interface, for several values of $\omega$ (see legend).

$$
\begin{aligned}
\gamma_{\text {stim }}\left(\mathbf{D}_{12}, \omega_{0}, z\right)= & \frac{2 \pi}{\hbar^{2}} \int d \omega\langle W(\omega)\rangle \\
& \times \frac{1}{2 \epsilon_{0}}\left|\mathbf{D}_{12} \cdot \mathbf{u}_{\mathbf{K}}(\mathbf{r})\right|^{2} \delta\left(\omega-\omega_{0}\right) .
\end{aligned}
$$

It follows that Einstein's coefficient for stimulated emission in mode $\mathbf{K}, B_{21}=\gamma_{\text {stim }}\left(\mathbf{D}_{12}, \omega_{0}, z\right) /\left\langle W\left(\omega_{0}\right)\right\rangle$, is given by

$$
B_{21}=\frac{\pi\left|\mathbf{D}_{12}\right|^{2}}{\epsilon_{0} \hbar^{2}} \frac{\exp \left(2 i \gamma_{1} z\right)}{L\left(\omega_{0}\right)}\left|d_{12, \|} \cos \phi-\frac{K}{\gamma_{1}} d_{12, z}\right|^{2},
$$

where $d_{12, \|}$ and $d_{12, z}$ are defined above, and $\phi$ is the angle between the projection of $\mathbf{D}_{12}$ on the interface and $\mathbf{K}$. When dealing with an isotropic distribution of dipoles, $B_{12}$ should be averaged over the directions of $\mathbf{D}_{12}$, in the same way as in Sec. V A 1. We get the total Einstein coefficient for stimulated emission in mode $\mathbf{K}$

$$
B_{21, \text { total }}=\frac{\pi\left|\mathbf{D}_{12}\right|^{2}}{3 \epsilon_{0} \hbar^{2}} \frac{\exp \left(2 i \gamma_{1} z\right)}{L\left(\omega_{0}\right)}\left[1-\epsilon\left(\omega_{0}\right)\right] .
$$

To describe the amplification of a surface-plasmon beam by an amplifying medium, it is interesting to derive the ratio $r\left(\omega_{0}, z\right)=A_{21}^{(i)}\left(\left|\mathbf{D}_{12}\right|, \omega_{0}, z\right) / B_{21, \text { total }}\left(\left|\mathbf{D}_{12}\right|, \omega_{0}, z\right), \quad$ where $A_{21}^{(i)}\left(\left|\mathbf{D}_{12}\right|, \omega_{0}, z\right)=\gamma_{\text {spont }}^{0}\left\langle F_{P, c l}^{(i)}\left(\mathbf{d}_{12}, \omega_{0}, z\right)\right\rangle$ stands for the total spontaneous emission rate of the dipole close to the interface [ $(i)$ denotes interface], and $\gamma_{\text {spont }}^{0}$ is given above. It can be computed with Eq. (24), using Green's tensor of a plane interface (this rate includes all the waves that can be emitted, not only surface plasmons). $\langle\cdot\rangle$ stands for average over the orientations $\mathbf{d}_{12}$ of the dipole. $r\left(\omega_{0}, z\right)$ gives the threshold energy per unit surface $W_{c}\left(\omega_{0}\right)$ at which the stimulated emission rate equals the spontaneous one. It can be written as

$$
r\left(\omega_{0}, z\right)=r^{0}\left(\omega_{0}\right) \frac{\left\langle F_{P, c l}^{(i)}\left(\mathbf{d}_{12}, \omega_{0}, z\right)\right\rangle}{\exp \left(2 i \gamma_{1} z\right)\left[1-\epsilon\left(\omega_{0}\right)\right]} L\left(\omega_{0}\right),
$$

where $r^{0}\left(\omega_{0}\right)=\frac{\hbar \omega^{3}}{\pi^{2} c^{3}}$ is the ratio of the Einstein's coefficients in a vacuum. Figure 4 shows $r(\omega, z)$ as a function of $\omega$. From 0 to approximately $35 \mathrm{~nm}$, the ratio decreases, mainly because the (total) spontaneous emission rate $A_{21}^{(i)}$ decreases. Above $35 \mathrm{~nm}$, the ratio increases as $B_{21}$ decreases because of the exponential decay of the surface-plasmon field away from this interface. Note that for a given frequency $\omega, r\left(\omega_{0}, z\right)$ rises at lower distances for $\omega$ closer to the surface-plasmon asymptote frequency.

These results can be used to calculate the amount of power that undergoes stimulated emission of surface plasmons in a gain medium in close vicinity of the silver interface. We consider a parallel beam of surface plasmons. and suppose that they are excited via a grating or a prism by a $\mathrm{He}-\mathrm{Ne}$ laser whose emission has a linewidth of about $\Delta \omega$ $=10 \mathrm{MHz}$ centered around $\omega_{0}=2 \mathrm{eV}$, and that they carry $P=1 \mathrm{~mW}$ of power per $\mu \mathrm{m}$. The spectral power at maximum, assuming a lorentzian profile, is given by $P_{\omega}\left(\omega_{0}\right)$ $=P / \pi \Delta \omega$, and the associated spectral energy per unit surface is $W\left(\omega_{0}\right)=P_{\omega}\left(\omega_{0}\right) / v_{g}=\frac{P}{\pi \Delta \omega v_{g}}$ where $v_{g}=\frac{d K}{d \omega}$ is the group velocity of surface plasmons, close to $c$ below the asymptote frequency. The spectral energy per unit surface at maximum of these surface plasmons is then $W\left(\omega_{0}\right) \approx \frac{P}{\pi \Delta \omega c}$ $\approx 10^{3} \hbar \mathrm{nm}^{2}$. This value is far above those of Fig. 4: stimulated emission in a freely propagating surface-plasmon beam is several orders of magnitude higher than spontaneous emission.

\section{CONCLUSION}

In this paper, we have extended previous work on quantization of surface plasmons by introducing a formalism that can use experimental values of the dielectric constant instead of using a specific model for the free-electron gas. The key step is the derivation of the energy of a surface plasmon in a dispersive nonlossy medium. The standard quantization scheme in Coulomb's gauge yields the quantum form of the field. This scheme can be extended in a straightforward way to thin metallic films. To illustrate the formalism, we have derived the spontaneous emission rate of surface plasmons by a two-level system placed close to an interface supporting surface waves as well as Einstein's coefficients. This quantized theory of surface plasmon will be useful to analyze specific quantum effects such as antibunching, single plasmon interference, quantum coherence properties, but also to derive the interactions of surface waves with other quantum objects, as quantum wells, for example.

\section{ACKNOWLEDGMENTS}

The authors acknowledge C'nano Ile-de-France and the French Agence Nationale de la Recherche (ANR) for financial support through the project LAPSUS. A.A. acknowledges financial support from the French Ministry of Defense through a grant from the Direction Générale de l'Armement (DGA).

\section{APPENDIX A: DIELECTRIC CONSTANT MODEL}

When cases with losses are considered in this paper, we must sometimes consider the case of complex frequencies. An analytical model for the dielectric constant is needed to evaluate $\epsilon(\omega)$ when $\omega$ is complex. The dielectric constant model which has been used in this paper is the following: 


$$
\epsilon(\omega)=\epsilon_{\infty}-\frac{\omega_{p}^{2}}{\omega^{2}+i \beta \omega}+i \frac{\sigma}{\epsilon_{0} \omega},
$$

where we take the values $\boldsymbol{\epsilon}_{\infty}=5, \hbar \omega_{p}=9.1 \mathrm{eV}$, and $\hbar \beta$ $=0.021 \mathrm{eV}$ of Drude's model given by Ref. 14 . We add also a conductivity term to have a better fit of the imaginary part of the dielectric constant, we take $\hbar \sigma / \epsilon_{0}=1.8 \mathrm{eV}$. Figure 5 compares the experimental data ${ }^{36}$ and both Drude's model from Ref. 14 and the model used in this paper in Eq. (A1).

\section{APPENDIX B: DERIVATION OF THE ENERGY}

In this section, we focus on the derivation of the electromagnetic energy associated with surface waves. The main idea is to derive the work done by an external operator to build adiabatically the field amplitude in a nonlossy medium. The energy balance from time $t=0$ to $t=T$ reads

$$
U=\int_{0}^{T} d t \int d^{3} \mathbf{r}\left[\mathbf{E} \frac{\partial \mathbf{D}}{\partial t}+\mathbf{H} \frac{\partial \mathbf{B}}{\partial t}\right]=\int_{0}^{T} d t \int d^{3} \mathbf{r}(-\mathbf{j} \cdot \mathbf{E})
$$

in which $\int_{0}^{T} d t \int d^{3} \mathbf{r}(-\mathbf{j} \cdot \mathbf{E})$ is the operator's work on the system between $t=0$ and $t=T$. $T$ must be large for this work to be adiabatic. $U$ then does not depend on $T$. In other words, this is also the total electromagnetic energy of the system. Due to the exponential decrease along the $z$ axis, the contribution of the Poynting vector, which should appear in the left term of Eq. (B1), drops to zero. We want hence to derive the first term of Eq. (B1) to obtain the electromagnetic energy.

The first step is to introduce time-dependent amplitudes in Eq. (6): $A_{\mathbf{K}}$ is then replaced by $A_{\mathbf{K}}(t)$ in which $A_{\mathbf{K}}(t)=A_{\mathbf{K}}$ $\times f(t)$. This accounts for operator's work. For the sake of convenience, we take a $2 T$-periodic function for which $f(0)$ $=f(2 T)=0$ and $f(T)=1$, so that we can write $f(t)$ $=\Sigma_{n} f_{n} \exp \left(i 2 \pi n \frac{t}{2 T}\right) . T$ is the typical time of variation in the amplitude $A_{\mathbf{K}}(t)$. $T$ has to be taken sufficiently large to consider the work done by the operator as adiabatic. [For instance, $f(t)=\sin \left(\pi \frac{t}{2 T}\right)$ ].

Lengthy and tedious calculation ${ }^{37}$ then leads to the following expression for $U$ :

$$
\begin{aligned}
U= & \sum_{\mathbf{K}} \epsilon_{0} S \omega^{2} \frac{1}{L(\omega)} \sum_{j=1,2} \frac{1}{2\left|\gamma_{j}\right|}\left[\left(1+\frac{K^{2}}{\left|\gamma_{j}\right|^{2}}\right) \frac{d\left[\omega \epsilon_{j}(\omega)\right]}{d \omega}\right. \\
& \left.+\left|\frac{\epsilon_{j}}{\gamma_{j}}\right|^{2} \frac{\omega^{2}}{c^{2}}\right]\left|A_{\mathbf{K}}\right|^{2} .
\end{aligned}
$$

We use the degree of freedom to set $L(\omega)$ as we wish, to drastically simplify this equation. We set

$$
L(\omega)=\frac{1}{2} \sum_{j=1,2} \frac{1}{2\left|\gamma_{j}\right|}\left[\left(1+\frac{K^{2}}{\left|\gamma_{j}\right|^{2}}\right) \frac{d\left[\omega \epsilon_{j}(\omega)\right]}{d \omega}+\left|\frac{\epsilon_{j}}{\gamma_{j}}\right|^{2} \frac{\omega^{2}}{c^{2}}\right]
$$

and put Eq. (B2) under the form

$$
U=\sum_{\mathbf{K}} \epsilon_{0} S \omega^{2} 2\left|A_{\mathbf{K}}\right|^{2}
$$

Let us give equivalent expressions ${ }^{37}$ of Eq. (B3). Using Eq. (4), the definition of $\gamma_{j}$, and $\epsilon(\omega) \leq-1$ at the frequencies of surface plasmon of a single interface, Eq. (B3) can be written

$$
L(\omega)=\frac{-\epsilon(\omega)}{2\left|\gamma_{1}\right|}+\frac{1}{4\left|\gamma_{2}\right|}\left[\frac{1-\epsilon(\omega)}{-\epsilon(\omega)} \frac{d[\omega \epsilon(\omega)]}{d \omega}-1-\epsilon(\omega)\right] .
$$

This is equivalent to the normalization condition

$$
\int_{-\infty}^{\infty} d z \frac{1}{2}\left[\frac{d\left[\omega \epsilon_{j}(\omega)\right]}{d \omega}+\left|\epsilon_{j}(\omega)\right| \frac{|1+\epsilon(\omega)|}{1+|\epsilon(\omega)|}\right]\left|\mathbf{u}_{\mathbf{K}}(z)\right|^{2}=1 .
$$

This equation can also be put in a more familiar form

$$
\int_{-\infty}^{\infty} d z\left[\frac{\epsilon_{0}}{2} \frac{d\left[\omega \epsilon_{j}(\omega)\right]}{d \omega}\left|\mathbf{E}_{\mathbf{K}}(z)\right|^{2}+\frac{1}{2 \mu_{0}}\left|\mathbf{B}_{\mathbf{K}}(z)\right|^{2}\right]=\epsilon_{0} \omega^{2}\left|A_{\mathbf{K}}\right|^{2},
$$

where $\mathbf{E}_{\mathbf{K}}(z)=i \omega A_{\mathbf{K}} \mathbf{u}_{\mathbf{K}}(z)$ and $\quad \mathbf{B}_{\mathbf{K}}(z)=i A_{\mathbf{K}} \mathbf{b}_{\mathbf{K}}(z) \quad$ so that $\mathbf{E}(\mathbf{r}, t)=\Sigma_{\mathbf{K}} \exp (i \mathbf{K} \cdot \mathbf{r}) \mathbf{E}_{\mathbf{K}}(z) \exp (-i \omega t)+$ c.c. and $\mathbf{B}(\mathbf{r}, t)$ $=\Sigma_{\mathbf{K}} \exp (i \mathbf{K} \cdot \mathbf{r}) \mathbf{B}_{\mathbf{K}}(z) \exp (-i \omega t)+$ c.c. $]$.

\section{APPENDIX C: QUANTUM CALCULATION OF THE SURFACE-PLASMON EMISSION RATE OF A DIPOLE}

We follow the same steps as in the derivation of the photon emission rate by a two-level system in a vacuum. The fundamental and excited states of the two-level system are denoted $|1\rangle$ and $|2\rangle$, respectively, associated with energies $E_{1}$ and $E_{2}$. We define a circular frequency $\omega_{0}$, so that $E_{2}-E_{1}$ $=\hbar \omega_{0}$. The two-level system is initially in its excited state, and there are $n_{\mathbf{K}}$ surface plasmons so that the global initial state can be written: $|i\rangle=\left|2, n_{\mathbf{K}}\right\rangle$. In the final state, the dipole is in its fundamental state and a surface plasmon has been created in a mode $\mathbf{K}$. We denote the final global state $|f\rangle$ $=\left|1, n_{\mathbf{K}}+1\right\rangle$. The interaction Hamiltonian is $-\hat{\mathbf{D}} \cdot \hat{\mathbf{E}}$, where $\hat{\mathbf{D}}$ is the electric-dipole moment operator and $\hat{\mathbf{E}}$ the quantum electric-field operator introduced in Sec. IV, at the position of the emitter. The emission rate (inverse of the lifetime $\tau$ of the excited state) is given by Fermi's golden rule

$$
\gamma=\frac{2 \pi}{\hbar} \sum_{f}|\langle f|\hat{\mathbf{D}} \cdot \hat{\mathbf{E}}| i\rangle|^{2} \delta\left(E_{2}-E_{1}-\hbar \omega\right) .
$$

This expression can be rewritten as a sum over the modes $\mathbf{K}$

$$
\gamma=\frac{2 \pi}{\hbar} \sum_{\mathbf{K}} M_{\mathbf{K}} \delta\left(E_{2}-E_{1}-\hbar \omega\right),
$$

where $M_{\mathbf{K}}=\left|\left\langle 1, n_{\mathbf{K}}+1|\hat{\mathbf{D}} \cdot \hat{\mathbf{E}}| 2, n_{\mathbf{K}}\right\rangle\right|^{2}$. We note $\langle 2|\hat{\mathbf{D}}| 1\rangle=\mathbf{D}_{12}$.

Using the former expression and Eqs. (13), (14), and (16), we obtain the following matrix element:

$$
M_{\mathbf{K}}=\frac{\hbar \omega}{2 \epsilon_{0} S}\left|\mathbf{D}_{12} \cdot \mathbf{u}_{1, \mathbf{K}}(z)\right|^{2}\left(n_{\mathbf{K}}+1\right) .
$$

In this equation, the $n_{\mathbf{K}}$ term stands for the stimulated emission and the constant term 1 accounts for the spontaneous emission. This section is devoted to the spontaneous emission so that we do not consider the term associated to $n_{\mathbf{K}}$. 


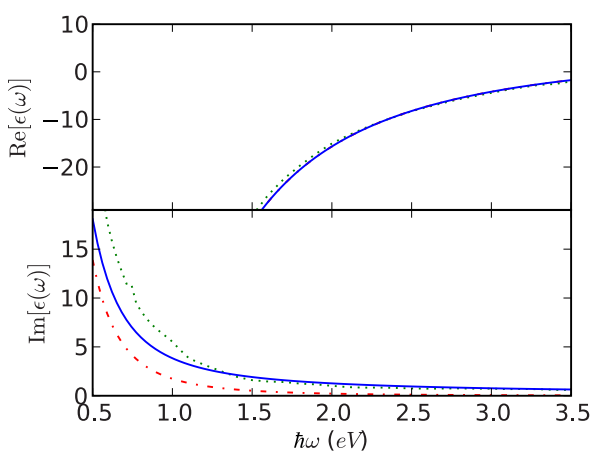

FIG. 5. (Color online) Real and imaginary parts of the dielectric constant for silver. Experimental data from Ref. 36 (dotted line), Drude's model from Ref. 14 (dashed-dotted line) and fit used in this paper (solid line) are plotted.

Using Eqs. (C2) and (C3), substituting a continuous sum over the vectors $\mathbf{K}$ in polar coordinates to the discrete sum $\Sigma_{\mathbf{K}}$, and now writing $\gamma_{\text {spont }}$ instead of $\gamma$ we get

$$
\begin{aligned}
\gamma_{\text {spont }}= & \frac{2 \pi}{\hbar} \int_{0}^{\infty} d K K \frac{S}{(2 \pi)^{2}} \frac{\hbar \omega}{2 \epsilon_{0} S} \delta\left(E_{2}-E_{1}-\hbar \omega\right) \\
& \times \int_{0}^{2 \pi} d \theta\left|\mathbf{D}_{12} \cdot \mathbf{u}_{1, \mathbf{K}}(z)\right|^{2} .
\end{aligned}
$$

The integration over the directions $\theta$ of $\mathbf{K}$ can now be performed using Eq. (5). We first write

$$
\begin{aligned}
\left|\mathbf{D}_{12} \cdot \mathbf{u}_{1, \mathbf{K}}(z)\right|^{2}= & \left|\mathbf{D}_{12}\right|^{2} \frac{1}{L\left(\omega_{s p}\right)} \exp \left(2 i \gamma_{j} z\right)\left[\left|\mathbf{d}_{12, \|} \cdot \hat{\mathbf{K}}\right|^{2}\right. \\
& \left.+\left|d_{12, z} \frac{K}{\gamma_{j}}\right|^{2}+2 \operatorname{Re}\left(\mathbf{d}_{12, \|} \cdot \hat{\mathbf{K}} d_{12, z}^{*} \frac{K}{\gamma_{j}^{*}}\right)\right] .
\end{aligned}
$$

The third term inside the square brackets on the right-hand side of Eq. (C5) vanishes after integration over $\theta$, as this term depends on $\theta$ only through $\hat{\mathbf{K}}$ and as $\int_{0}^{2 \pi} d \theta \hat{\mathbf{K}}=\mathbf{0}$. The integration thus gives

$$
\int_{0}^{2 \pi} d \theta\left|\mathbf{D}_{12} \cdot \mathbf{u}_{1, \mathbf{K}}(z)\right|^{2}=\frac{2 \pi}{L_{e f f}\left(z, d_{12}, \omega_{0}\right)}\left|\mathbf{D}_{12}\right|^{2} .
$$

$L_{e f f}\left(z, d_{12}, \omega_{0}\right)$ is defined by Eq. (19). The spontaneous emission rate of surface plasmon can then be cast in the form given by Eq. (18).

Averaging $\gamma_{\text {spont }}$ over all the possible directions $(\theta, \phi)$ and all the precession angles $\psi$ of $\mathbf{D}_{12}$, we get the "total" spontaneous emission rate of surface plasmons

$$
\begin{aligned}
\left.\gamma_{\text {spont,total }}\left|\mathbf{D}_{12}\right|, \omega_{0}, z\right)= & \frac{1}{8 \pi^{2}} \int_{0}^{\pi} d \theta \sin \theta \int_{0}^{2 \pi} d \phi \int_{0}^{2 \pi} d \psi \\
& \times \gamma_{\text {spont }}\left[\mathbf{R}_{\theta, \phi, \psi}\left(\mathbf{D}_{12}\right), \omega_{0}, z\right], \quad(\mathrm{C} 7)
\end{aligned}
$$

where $\mathbf{R}_{\theta, \phi, \psi}\left(\mathbf{D}_{12}\right)$ is $\mathbf{D}_{12}$ rotated by Euler's angles for nutation, precession and intrinsic rotation $\theta, \phi$, and $\psi$, respectively. The result of the integration over $\theta, \phi$, and $\psi$ is given by Eq. (23).

\section{APPENDIX D: DERIVATION OF THE EMISSION RATE OF A DIPOLE IN THE CLASSICAL LOSSY CASE}

The aim of this section is to derive an explicit form of the Purcell factor due to the presence of surface plasmons by using the Green's tensor approach. Using Ref. 35, one can write the surface-plasmon contribution to the Green's tensor evaluated at the position of the source $\mathbf{r}$

$$
\overleftrightarrow{\mathbf{G}}_{s p}(\mathbf{r}, \mathbf{r}, \omega)=\int \frac{d^{2} \mathbf{K}}{(2 \pi)^{2}}\left[\frac{\stackrel{\mathbf{f}}{\mathbf{f}}(\mathbf{K}, z, z)}{\omega-\omega_{s p}}-\frac{\stackrel{\leftrightarrow}{\mathbf{f}^{*}}(-\mathbf{K}, z, z)}{\omega+\omega_{s p}^{*}}\right],
$$

where $\overleftrightarrow{\mathbf{f}}(\mathbf{K}, z, z)$ is given by

$$
\overleftrightarrow{\mathbf{f}}(\mathbf{K}, z, z)=-g\left(K, \omega_{s p}\right)\left(\hat{\mathbf{K}}-\frac{K}{\gamma_{1}} \hat{\mathbf{z}}\right)\left(\hat{\mathbf{K}}-\frac{K}{\gamma_{1}} \hat{\mathbf{z}}\right) \exp \left(2 i \gamma_{1} z\right)
$$

as $z>0$, with $g\left(K, \omega_{s p}\right)=\frac{c^{2} \gamma_{1}^{2} \epsilon\left(\omega_{s p}\right)}{\omega_{s p}^{2}} R\left(K, \omega_{s p}\right)$ and $R^{-1}\left(K, \omega_{s p}\right)$ $=-i \frac{\partial}{\partial \omega}\left[\gamma_{1}(K, \omega) \epsilon(\omega)-\gamma_{2}(K, \omega)\right]_{\omega=\omega_{s p}}$. Injecting Eq. (D1) in Eq. (24), we obtain

$$
\begin{aligned}
F_{P, c l}\left(\mathbf{d}_{12}, \omega, z\right)= & \frac{6 \pi c}{\omega} \int \frac{d^{2} \mathbf{K}}{(2 \pi)^{2}} \operatorname{Im}\left[\frac{\mathbf{d}_{12}^{*} \cdot \overleftrightarrow{\mathbf{f}}(\mathbf{K}, z, z) \mathbf{d}_{12}}{\omega-\omega_{s p}}\right. \\
& \left.-\frac{\mathbf{d}_{12}^{*} \cdot \stackrel{\mathbf{f}^{*}}{ }(-\mathbf{K}, z, z) \mathbf{d}_{12}}{\omega+\omega_{s p}^{*}}\right] .
\end{aligned}
$$

Writing

$F_{\mathbf{K}}\left(\mathbf{d}_{12}, \omega, z\right)=\mathbf{d}_{12}^{*} \cdot \stackrel{\leftrightarrow}{\mathbf{f}}(\mathbf{K}, z, z) \mathbf{d}_{12}=F_{\mathbf{K}}^{\prime}\left(\mathbf{d}_{12}, \omega, z\right)+i F_{\mathbf{K}}^{\prime \prime}\left(\mathbf{d}_{12}, \omega, z\right)$ and

$$
\begin{aligned}
& \rho_{\omega_{s p}^{\prime}}^{\prime}(\omega)=\frac{\omega-\omega_{s p}^{\prime}}{\left(\omega-\omega_{s p}^{\prime}\right)^{2}+\omega_{s p}^{\prime \prime}}, \\
& \rho_{\omega_{s p}^{\prime \prime}}^{\prime \prime}(\omega)=\frac{\omega_{s p}^{\prime \prime}}{\left(\omega-\omega_{s p}^{\prime}\right)^{2}+\omega_{s p}^{\prime \prime 2}},
\end{aligned}
$$

we find

$$
\begin{aligned}
F_{P, c l}\left(\mathbf{d}_{12}, \omega, z\right)= & \frac{6 \pi c}{\omega} \int \frac{d^{2} \mathbf{K}}{(2 \pi)^{2}}\left[F_{\mathbf{K}}^{\prime}\left(\mathbf{d}_{12}, \omega, z\right) \rho_{\omega_{s p}}^{\prime \prime}(\omega)\right. \\
& +F_{\mathbf{K}}^{\prime \prime}\left(\mathbf{d}_{12}, \omega, z\right) \rho_{\omega_{s p}}^{\prime}(\omega) \\
& -F_{\mathbf{K}}^{\prime}\left(\mathbf{d}_{12}, \omega, z\right) \rho_{-\omega_{s p}^{*}}^{\prime \prime}(\omega) \\
& \left.+F_{\mathbf{K}}^{\prime \prime}\left(\mathbf{d}_{12}, \omega, z\right) \rho_{-\omega_{s p}^{*}}^{\prime}(\omega)\right] .
\end{aligned}
$$

This expression is to be compared to Eq. (C2) in the nonlossy case. Both expressions are written as sums over the modes $\mathbf{K}$, of the contribution of each mode to the spontaneous emission rate. When losses are low, the last two terms on the right-hand side of Eq. (D4) are antiresonant, as $\rho_{-\omega_{s p}^{*}}^{\prime \prime}(\omega)$ and $\rho_{-\omega_{s p}^{*}}^{\prime}(\omega)$ are centered around $-\omega_{s p}^{\prime}$. The second term has also a small contribution as the average value of $\rho_{\omega_{s p}}^{\prime}(\omega)$ is 0 . The main contribution comes from the first term, as $\rho_{\omega_{s p}}^{\prime \prime}(\omega)$ goes to $-i \pi \delta\left(\omega-\omega_{s p}\right)$ in the nonlossy limit. Its extremum 
value is $\frac{-2 Q}{\omega_{s p}^{\prime}}$. Starting again from Eq. (D1), we write

$$
\begin{aligned}
\overleftrightarrow{\mathbf{G}}_{s p}(\mathbf{r}, \mathbf{r}, \omega)= & \frac{1}{(2 \pi)^{2}} \int_{0}^{\infty} d K K\left\{\frac{1}{\omega-\omega_{s p}} \int_{0}^{2 \pi} d \theta[\overleftrightarrow{\mathbf{f}}(\mathbf{K}, z, z)]\right. \\
& \left.-\frac{1}{\omega+\omega_{s p}^{*}} \int_{0}^{2 \pi} d \theta\left[\overleftrightarrow{\mathbf{f}^{*}}(-\mathbf{K}, z, z)\right]\right\}, \quad
\end{aligned}
$$

aiming at performing the integral over the directions $\theta$ of $\mathbf{K}$. We first calculate

$$
\int_{0}^{2 \pi} d \theta[\overleftrightarrow{\mathbf{f}}(\mathbf{K}, z, z)]=-2 \pi g\left(K, \omega_{s p}\right) \overleftrightarrow{\mathbf{U}}\left(z, \omega_{s p}\right)
$$

where $\overleftrightarrow{\mathbf{U}}\left(z, \omega_{s p}\right)=\exp \left(2 i \gamma_{1} z\right)\left[\frac{1}{2}(\hat{\mathbf{x}} \hat{\mathbf{x}}+\hat{\mathbf{y}} \hat{\mathbf{y}})-\epsilon\left(\omega_{s p}\right) \hat{\mathbf{z}} \hat{\mathbf{z}}\right]$. Inserting Eq. (D6) in Eq. (D5) yields

$$
\begin{aligned}
\overleftrightarrow{\mathbf{G}}_{s p}(\mathbf{r}, \mathbf{r}, \omega)= & \frac{1}{2 \pi} \int_{0}^{\infty} d K K\left[\frac{-g\left(K, \omega_{s p}\right)}{\omega-\omega_{s p}} \overleftrightarrow{\mathbf{U}}\left(z, \omega_{s p}\right)\right. \\
& \left.+\frac{g^{*}\left(K, \omega_{s p}\right)}{\omega+\omega_{s p}^{*}} \overleftrightarrow{\mathbf{U}}^{*}\left(z, \omega_{s p}\right)\right]
\end{aligned}
$$

Inserting Eq. (D7) in Eq. (24), one finds

$$
\begin{aligned}
F_{P, c l}\left(\mathbf{d}_{12}, \omega_{0}, z\right)= & \frac{3 c}{\omega_{0}} \int_{0}^{\infty} d K K \operatorname{Im} \\
& \times\left[\frac{-g\left(K, \omega_{s p}\right)}{\omega_{0}-\omega_{s p}} \mathbf{d}_{12}^{*} \cdot \stackrel{\leftrightarrow}{\mathbf{U}}\left(z, \omega_{s p}\right) \mathbf{d}_{12}\right. \\
& \left.+\frac{g^{*}\left(K, \omega_{s p}\right)}{\omega_{0}+\omega_{s p}^{*}} \mathbf{d}_{12} \cdot \stackrel{\leftrightarrow}{\mathbf{U}}^{*}\left(z, \omega_{s p}\right) \mathbf{d}_{12}^{*}\right]
\end{aligned}
$$

with $\quad \mathbf{d}_{12}^{*} \cdot \overleftrightarrow{\mathbf{U}}\left(z, \omega_{s p}\right) \mathbf{d}_{12}=\exp \left(2 i \gamma_{1} z\right)\left[\frac{1}{2}\left|\mathbf{d}_{12, \|,}\right|^{2}-\epsilon\left(\omega_{s p}\right)\left|d_{12, z}\right|^{2}\right]$. In the nonlossy case, $g\left(K, \omega_{s p}\right)$ and $\mathbf{d}_{12}^{*} \cdot \overleftrightarrow{\mathbf{U}}\left(z, \omega_{s p}\right) \mathbf{d}_{12}$ are real, and $\operatorname{Im} \frac{1}{\omega-\omega_{s p}}$ goes to ${ }^{42}-i \pi \delta\left(\omega-\omega_{s p}\right)$. Hence, assuming $\omega$ $>0$ and using the expressions of $\mathbf{d}_{12}^{*} \cdot \overleftrightarrow{\mathbf{U}}\left(z, \omega_{s p}\right) \mathbf{d}_{12}$ and $g\left(K, \omega_{s p}\right)$ given above, one gets Eq. $(25)$.
${ }^{1}$ R. Loudon, The Quantum Theory of Light, 3rd ed. (Oxford University Press, New York, 2000).

${ }^{2}$ H. Raether, Surface Plasmons on Smooth and Rough Surfaces and on Gratings (Springer-Verlag, Berlin, 1988).

${ }^{3}$ C. J. Powell and J. B. Swan, Phys. Rev. 115, 869 (1959).

${ }^{4}$ A. V. Akimov, A. Mukherjee, C. L. Yu, D. E. Chang, A. S. Zibrov, P. R. Hemmer, H. Park, and M. D. Lukin, Nature (London) 450, 402 (2007).

${ }^{5}$ R. Kolesov, B. Grotz, G. Balasubramanian, R. J. Stohr, A. A. L. Nicolet, P. R. Hemmer, F. Jelezko, and J. Wrachtrup, Nat. Phys. 5, 470 (2009).

${ }^{6}$ A. Y. Smuk and N. M. Lawandy, Appl. Phys. B: Lasers Opt. 84, 125 (2006).

${ }^{7}$ K. Li, X. Li, M. I. Stockman, and D. J. Bergman, Phys. Rev. B 71, 115409 (2005).

${ }^{8}$ D. J. Bergman and M. I. Stockman, Phys. Rev. Lett. 90, 027402 (2003).

${ }^{9}$ M. A. Noginov, G. Zhu, M. Bahoura, J. Adegoke, C. Small, B. A. Ritzo, V. P. Drachev, and V. M. Shalaev, Appl. Phys. B: Lasers Opt. 86, 455 (2007).

${ }^{10}$ J. Seidel, S. Grafstrom, and L. Eng, Phys. Rev. Lett. 94, 177401 (2005).

${ }^{11}$ M. Ambati, S. H. Nam, E. Ulin-Avila, D. A. Genov, G. Bartal, and X. Zhang, Nano Lett. 8, 3998 (2008).

${ }^{12}$ M. A. Noginov, G. Zhu, M. Mayy, B. A. Ritzo, N. Noginova, and V. A. Podolskiy, Phys. Rev. Lett. 101, 226806 (2008).

${ }^{13}$ I. De Leon and P. Berini, Phys. Rev. B 78, 161401(R) (2008).

${ }^{14}$ M. Ambati, D. A. Genov, R. F. Oulton, and X. Zhang, IEEE J. Sel. Top. Quantum Electron. 14, 1395 (2008).

${ }^{15}$ K. F. MacDonald, Z. L. Samson, M. I. Stockman, and N. I. Zheludev, Nat. Photonics 3, 55 (2009).

${ }^{16}$ L. Cao and M. L. Brongersma, Nat. Photonics 3, 12 (2009).

${ }^{17}$ I. E. Protsenko, A. V. Uskov, O. A. Zaimidoroga, V. N. Sam- oilov, and E. P. O’Reilly, Phys. Rev. A 71, 063812 (2005).

${ }^{18}$ D. J. Bergman and M. I. Stockman, Laser Phys. 14, 409 (2004).

${ }^{19}$ N. I. Zheludev, S. L. Prosvirnin, N. Papasimakis, and V. A. Fedotov, Nat. Photonics 2, 351 (2008).

${ }^{20}$ M. A. Noginov, G. Zhu, A. M. Belgrave, R. Bakker, V. M. Shalaev, E. E. Narimanov, S. Stout, E. Herz, T. Suteewong, and U. Wiesner, Nature (London) 460, 1110 (2009).

${ }^{21}$ R. F. Oulton, V. J. Sorger, T. Zentgraf, R.-M. Ma, C. Gladden, L. Dai, G. Bartal, and X. Zhang, Nature (London) 461, 629 (2009).

${ }^{22}$ W. L. Barnes, G. Bjork, J. M. Gerard, P. Jonsson, J. A. E. Wasey, P. T. Worthing, and V. Zwiller, Eur. Phys. J. D 18, 197 (2002).

${ }^{23}$ S. Fasel, M. Halder, N. Gisin, and H. Zbinden, New J. Phys. 8, 13 (2006).

${ }^{24}$ D. E. Chang, A. S. Sorensen, P. R. Hemmer, and M. D. Lukin, Phys. Rev. Lett. 97, 053002 (2006).

${ }^{25}$ Y. Gong and J. Vuckovic, Appl. Phys. Lett. 90, 033113 (2007).

${ }^{26}$ E. Altewischer, M. P. van Exter, and J. P. Woerdman, Nature (London) 418, 304 (2002).

${ }^{27}$ E. Moreno, F. J. Garcia-Vidal, D. Erni, J. I. Cirac, and L. MartinMoreno, Phys. Rev. Lett. 92, 236801 (2004).

${ }^{28}$ S. Fasel, F. Robin, E. Moreno, D. Erni, N. Gisin, and H. Zbinden, Phys. Rev. Lett. 94, 110501 (2005).

${ }^{29}$ M. S. Tame, C. Lee, J. Lee, D. Ballester, M. Paternostro, A. V. Zayats, and M. S. Kim, Phys. Rev. Lett. 101, 190504 (2008).

${ }^{30}$ J. M. Elson and R. H. Ritchie, Phys. Rev. B 4, 4129 (1971).

${ }^{31}$ T. Gruner and D. G. Welsch, Phys. Rev. A 53, 1818 (1996).

${ }^{32}$ M. Babiker, N. C. Constantinou, and B. K. Ridley, Phys. Rev. B 48, 2236 (1993).

${ }^{33}$ S. Stallinga, Phys. Rev. E 73, 026606 (2006).

${ }^{34}$ L. Landau and E. Lifchitz, Electrodynamics of Continuous Media, 2nd ed. (Pergamon, Oxford, 1984).

${ }^{35}$ A. Archambault, T. V. Teperik, F. Marquier, and J. J. Greffet, Phys. Rev. B 79, 195414 (2009). 
${ }^{36}$ E. D. Palik, Handbook of Optical Constants of Solids (Academic, San Diego, 1985).

${ }^{37}$ See supplementary material at http://link.aps.org/supplemental/ 10.1103/PhysRevB.82.035411 for a detailed derivation of the energy of the surface-plasmon field, and calculation proving the equality between Eqs. (20) and (25).

${ }^{38}$ J. D. Jackson, Classical Electrodynamics, 3rd ed. (Wiley, New
York, 1999).

${ }^{39}$ L. Brillouin, Wave Propagation and Group Velocity (Academic Press, New York, 1960).

${ }^{40}$ G. W. Ford and W. H. Weber, Phys. Rep. 113, 195 (1984).

${ }^{41}$ E. M. Purcell, Phys. Rev. 69, 37 (1946).

${ }^{42}$ L. Novotny and B. Hecht, Principles of Nano-Optics (Cambridge University Press, New York, 2006). 